\title{
Soil Physical Properties as Influenced with Protected and Conventional Vegetable Production System in District Kangra of Himachal Pradesh
}

\author{
Shabnam* and Sanjay K. Sharma \\ Department of Soil Science, CSKHPKV, Palampur Himachal Pradesh, India \\ *Corresponding author
}

\section{A B S T R A C T}

Keywords

Protected, conventional and vegetables

Article Info

Accepted:

04 October 2018

Available Online:

10 November 2018
Protected cultivation is gaining momentum especially for vegetable cultivation in Himachal Pradesh. Therefore the present study was undertaken to assess the soil quality inside polyhouses vis a vis under conventional cultivation so as to ascertain the effect of intensive cropping and the management practices being followed by farmers under these two systems on soil physical properties. The main objective of this study was to assess the effect of conventional and protected systems of farming on soil physical parameters. Texture across the various sites varied from sandy loam to clay loam. Mean of two years (2015 and 2016) revealed comparatively lower bulk density inside protected cultivation $\left(1.12 \mathrm{Mg} \mathrm{m}^{-3}\right)$ as compared to conventional system $\left(1.24 \mathrm{Mg} \mathrm{m}^{-3}\right)$. There were as such no differences were observed in particle density. However, porosity and water holding capacity differ inside protected and conventional system of production. Considering overall mean of mean weight diameter (MWD) it was observed higher inside protected system $(1.49 \mathrm{~mm})$ as compared to conventional system $(1.40 \mathrm{~mm})$.

\section{Introduction}

Productivity and the sustainability of any production system not only depend upon the management practices but also on the environment as well as on the soil quality. An agricultural soil with good quality promotes and sustains good agricultural productivity with less environmental impact and possesses utmost physical, chemical and biological attributes to fulfill these requirements (Reynolds et al., 2009). Soil quality has been defined as "the capacity of specific kind of soil to function within ecosystem and land use boundaries to sustain biological productivity, maintain environmental quality and sustain plant, animal and human health" (Doran and Parkin, 1994). To assess the soil quality we have to consider various physical, chemical and biological attributes referred to as indicators. These indicators may directly monitor the soil or monitor the outcomes that are affected by the soil. India is the second largest producer of vegetables in the world, next to China. It produces 167.1 million tonnes of vegetables from an area of 9.5 million ha (NHB, 2015-16) which is however, much less than the actual requirement for providing balanced diet to every individual. To cope up with the burgeoning population, 
the total production of vegetables in India needs to be raised to at least 250 million tonnes by 2024-25. It means we have to increase the productivity vertically from the limited land resources as the per capita land availability is decreasing. In general, the farmers are still practicing less intensive and remunerative farming system under open field cultivation (Kokate et al., 2012). Besides, there are many constraints with respect to climatic conditions viz., moisture, temperature, sunshine hours, wind velocities, humidity and weather vagaries, coupled with nutrient deficiencies, excessive weed growth and insect pests attack leading to poor productivity. To overcome these constraints, "protected cultivation" a specialized high-tech cultivation system under the polyhouses is being emphasized for the last two decades. Protected vegetable cultivation has proven to be a good farming practice in various parts of different countries and has been developed rapidly during recent years because of the comparatively higher economic benefits

In Himachal Pradesh too, protected vegetable cultivation has gained quite a good momentum particularly, for vegetables and commercial crops due to higher productivity, intensive cropping and assured income. The state government is also emphasizing protected farming through Horticultural Technology Mission and Pandit Deen Dayal Kisan Bagwan Samridhi Yojna. A large number of polyhouses has been constructed in the state occupying an area of about 223.2 ha (Chaudhary, 2016) and the indeterminate varieties of tomato, coloured capsicum and cucumber etc. have particularly emerged more popular among the farmers. The principle of protected cultivation is optimum utilization of inner space and to harness the off-season advantage. However, intensive cultivation coupled with very high use of specific inputs under protected conditions may prove detrimental to soil and produce quality in the long run due to over exploitation of native reserves of the nutrients. Sustainability of intensive agriculture system is linked to maintenance or enhancement of soil quality (Benbi and Saroa, 2012). The intensive use of chemical fertilizers and other inputs, air, moisture and temperature manipulations and higher productivity vis a vis nutrient removal under protected cultivation may however, affect the physical, chemical and biological properties of soil i.e. 'the soil quality'.

In the present scenario of vegetable production under protected conditions in Himachal Pradesh, there is intensive cultivation of vegetables and it becomes imperative to assess the impact of prevalent management practices with respect to use of fertilizers and other inputs on soil quality for sustained production. The changes in soil quality indicate, whether the management practices being adopted are sustainable or not. Owing to vast differences in management practices, growth conditions and the productivity of the vegetables, the differences in soil quality may be expected between the protected and open field cultivation.

\section{Materials and Methods}

The study sites were located in between $31^{\circ} 59.700^{\prime} \mathrm{N}$ and $76^{\circ} 39.033^{\prime} \mathrm{E}$ to $32^{\circ} 08.500$ $\mathrm{N}$ and $76^{\circ} 25.010^{\prime} \mathrm{E}$. For the present investigation twenty five farmers (five farmers per block) growing vegetables for at least five years under the protected (polyhouse) as well as conventional (open conditions) from Kangra district were selected randomly from five blocks viz; Baijnath, Bhedu-Mahadev, Bhawarna, Kangra and Dharamshala. The details of the selected sites are depicted in figure.1. After this a general survey of the selected farmers was carried out for assortment of necessary information about cultivation practices, cropping patterns and problems prevailing in the polyhouses 
growing vegetables. Capsicum was the predominant crop in most of the polyhouses. However cucumber, tomato, beans, coriander and cauliflower were also grown in some polyhouses. All the polyhouses selected for present study were naturally ventilated. These polyhouse didn't have any environmental control system except for the provision of adequate ventilation and fogger system to prevent basically the damage of weather aberrations and other natural agents.

\section{Results and Discussion}

\section{Physical parameters}

The physical indicators for assessing soil quality comprised texture, bulk density (BD), particle density (PD), porosity, water holding capacity and water stable aggregates of soils.

\section{Soil texture}

Soil texture refers to weight percentage of sand $(0.05$ to $2 \mathrm{~mm})$, silt $(0.002$ to $0.05 \mathrm{~mm})$ and clay $(<0.002 \mathrm{~mm})$. Soil texture is one of the most stable attributes of the soil which can only be modified slightly by the practices that cause mixing of different layers. Soil texture has an important effect on water and nutrient holding capacity. In general, sandy loam to loam texture is considered good for optimum crop growth because such soils allow easy movement of water, air and nutrients. In the present study soil texture under different sites varied from sandy loam to clay loam; however sandy loam was the most dominant texture under protected and conventional vegetable cultivation (Table 2). Chander et al., (2014) also reported almost similar status of soil texture in vegetable growing soils of subhumid and wet-temperate zones of Himachal Pradesh. Sand, silt and clay contents under protected system in the Kangra district ranged from 43 to 62,16 to 36 and 12 to 34 per cent, respectively. While for conventional vegetable production system sand, silt and clay varied from 41 to 60,16 to 36 and 15 to 34 per cent, respectively. Because texture does not change much therefore, samples for this parameter were analysed only once i.e. those collected during 2015. Comparatively higher sand percentage under protected conditions at some sites (Nora, Suri, Bodda, Arla-Khas, Samloti, Tang and Lower-Bagli) might be due to addition of sand by the farmers to alter texture, for making it more suitable for vegetable production.

\section{Bulk Density (BD)}

Generally, bulk density increases with increasing sand and rock content and decreases with addition of organic matter. The roots grow well in soils with low bulk densities whereas root growth begins to decline significantly at bulk density above $1.70 \mathrm{Mg} \mathrm{m}^{-3}$. Data in Table 3 revealed only slight changes in bulk density during the study period under two different management practices (protected and conventional). Bulk density during 2015 varied from 1.09 to 1.37 $\mathrm{Mg} \mathrm{m}^{-3}$ under protected environment, and for the same year bulk density under open environment ranges from 1.13 to $1.42 \mathrm{Mg} \mathrm{m}^{-3}$. Average bulk density for same year was found comparatively lower $\left(1.21 \pm 0.06 \mathrm{Mg} \mathrm{m}^{-3}\right)$ under protected system compared to conventional system $\left(1.24 \pm 0.05 \mathrm{Mg} \mathrm{m}^{-3}\right)$. However not much change in overall bulk density was observed both, under protected as well as conventional system during 2016 in comparison to 2015. Overall average bulk density during 2016 varied from 1.08 to 1.36 $\mathrm{Mg} \mathrm{m}^{-3}$ under protected system, while under conventional system it ranged from 1.15 to $1.41 \mathrm{Mg} \mathrm{m}^{-3}$. Based on the mean values of two years, it further revealed that among different blocks under protected system of vegetable production, Baijnath and Bhedu-Mahadev recorded higher average bulk density (1.22 $\mathrm{Mg} \mathrm{m}^{-3}$ ) while, Dharamshala block recorded 
the lowest average bulk density $\left(1.19 \mathrm{Mg} \mathrm{m}^{-3}\right)$. Conventional vegetable production system had average bulk density values of $1.27,1.26$, 1.24, 1.22 and $1.23 \mathrm{Mg} \mathrm{m}^{-3}$ at Baijnath, Bhedu-Mahadev, Bhawarna, Kangra and Dharamshala blocks, respectively. Similar range of bulk density was also observed by Kyandiah (2012) for soils of Himachal Pradesh. In general, if values of bulk density are less than $1.50 \mathrm{Mg} \mathrm{m}^{-3}$, then it is taken as low. Since most of the sites had low bulk density, the soils were less compact and therefore, good for the production of vegetables. Most of the sites under conventional vegetable production had higher bulk density values as compared to protected conditions which might be attributed to higher organic carbon contents observed under protected field conditions the role of intensive management (tillage operations, frequent applications of higher amount of organic manures and chemical fertilizers) and consequently better microbial activities and soil aggregations are the other reasons for the lower values of bulk density under protected environment as compared to open one as observed in the present study.

Farmers added more organic matter (FYM, vermicompost) inside polyhouse condition in comparison to open field conditions at most of the sites which might be also one of the factors for lower bulk density values under protected conditions. Herencia et al., (2011) also reported a decrease in bulk density in sites where practice of addition of FYM has been done under protected and conventional production systems.

\section{Particle Density (PD)}

The data on the status of particle density under protected and conventional conditions have been enumerated in Table 4. Though the particle density is considered one of the static properties, the samples collected during second year (2016), and were analysed for this property just to confirm the results for the previous year. As expected, no such variation was observed in particle density values for each of the locations under protected as well as conventional systems of agriculture. The mean particle density of two years among different sites varied between 2.11 to $2.52 \mathrm{Mg}$ $\mathrm{m}^{-3}$ under protected system of vegetable production with overall mean of $2.37 \pm 0.11$ $\mathrm{Mg} \mathrm{m}^{-3}$. Among different blocks which were selected for the present study under protected system of vegetable production lowest particle density was observed in Baijnath $\left(2.28 \mathrm{Mg} \mathrm{m}^{-}\right.$ ${ }^{3}$ ) followed by Bhawarna $\left(2.34 \mathrm{Mg} \mathrm{m}^{-3}\right)$ and Dharamshala blocks $\left(2.39 \mathrm{Mg} \mathrm{m}^{-3}\right)$. BheduMahadev soils had higher particle density among all the blocks $\left(2.42 \mathrm{Mg} \mathrm{m}^{-3}\right)$.

Average particle density under conventional system of vegetable production varied from 2.14 to $2.51 \mathrm{Mg} \mathrm{m}^{-3}$ with overall mean of all locations was $2.37 \pm 0.10 \mathrm{Mg} \mathrm{m}^{-3}$. The mean PD of all locations in a block was observed lowest in Baijnath $\left(2.31 \mathrm{Mg} \mathrm{m}^{-3}\right)$ followed by Bhawarna (2.35 $\mathrm{Mg} \mathrm{m}^{-3}$ ) and Kangra (2.38 $\mathrm{Mg} \mathrm{m}^{-3}$ ), while the highest was recorded for Dharamshala block $\left(2.41 \mathrm{Mg} \mathrm{m}^{-3}\right)$. The differences in particle density among various locations irrespective of the cultivation conditions might be due to differences in the parent material. Slightly lower values of particle density under protected condition might be due to binding of organic matter on various separates of soil. The reason behind slight variation in particle density under protected and conventional conditions may also be attributed to the management practices followed by farmers (Hillel, 1980).

\section{Porosity}

Porosity is the ratio of total volume of pore spaces to the total soil column volume and is an index of relative pore volume in a soil. 
The status of soil porosity for different sites under protected and conventional conditions is presented in the Table 5. Data depicted that during 2015 porosity ranged from 40.4 to 55.1 per cent under protected conditions, while it varied from 40.3 to 53.9 per cent under conventional vegetable production system with overall mean values across all the locations as $48.8 \pm 3$ and $47.4 \pm 2.9$ per cent, respectively. Very slight variations were observed in porosity during 2016 over the values computed during 2015 among different sites both under protected as well as conventional system of vegetable production.

Among different blocks selected for present study under protected system of vegetable production, the highest mean porosity in a block as a whole, was observed in Kangra block $(50.2 \%)$ while, the lowest in Baijnath (46.4\%). Similarly, mean porosity among different blocks as a whole under conventional production of vegetables was worked out to 45, 47.8, 47.1, 48.6 and 49 per cent for Baijnath, Bhedu-Mahadev, Bhawarna, Kangra and Dharamshala blocks, respectively. Overall mean porosity values were higher under protected conditions during both the years.

Since most of the polyhouse's surface soil exhibited low bulk and particle densities in comparison to samples from conventionally cultivated fields therefore, comparatively higher porosity values under polyhouse are as per expectation.

\section{Water Holding Capacity (WHC)}

Data pertaining to water holding capacity are given in Table 6. Perusal of data in Table 6 revealed average water holding capacity for both the years was higher under protected environment as compared to conventional system of vegetables production. It varied between 46.2 to 60.3 per cent during 2015 and between 47.2 to 60.2 per cent during 2016 under the protected conditions. The overall mean of all the locations during 2015 and 2016 for water holding capacity were $53.6 \pm 3.2$ and $54 \pm 3$ per cent, respectively. Among various sites under protected system highest average water holding capacity was found in Bhedu-Mahadev block (54.8\%) followed by Kangra (54.4\%) while the lowest was recorded at Baijnath (52.8\%).

Under conventional system of vegetable production, water holding capacity across different sites varied from 44.1 to 58.4 per cent and 44.3 to 56.2 per cent during 2015 and 2016, respectively. While, the overall mean water holding capacity across all the locations was $51.4 \pm 3.1$ and $51.3 \pm 2.8$ per cent for 2015 and 2016, respectively.

Table.1 Methods used for analysis of physical parameters

\begin{tabular}{|l|l|l|}
\hline Parameter & Method employed & Reference \\
\hline Texture & International pipette method & Piper (1966) \\
\hline Water holding capacity & Keen box method & Piper (1950) \\
\hline Bulk density & Core sampler method & Blake and Hartge (1986) \\
\hline Particle Density & Pycnometer method & $\begin{array}{l}\text { Gupta and Dhakshinamoorthy } \\
(1980)\end{array}$ \\
\hline Porosity & Empirical method & $\begin{array}{l}\text { Gupta and Dhakshinamoorthy } \\
(1980)\end{array}$ \\
\hline Aggregate analysis & Wet sieving method & Yoder (1936) \\
\hline
\end{tabular}


Table.2 Mechanical separates (\%) and soil texture under protected and conventional cultivation in district Kangra

\begin{tabular}{|c|c|c|c|c|c|c|c|c|c|}
\hline \multirow{2}{*}{$\begin{array}{l}\text { Sr. } \\
\text { No. }\end{array}$} & \multirow[t]{2}{*}{ Sites } & \multicolumn{4}{|c|}{ Protected } & \multicolumn{4}{|c|}{ Conventional } \\
\hline & & Sand & Silt & Clay & Texture & Sand & Silt & Clay & Texture \\
\hline \multicolumn{10}{|c|}{ Baijnath } \\
\hline 1. & Vikasnagar & 48 & 24 & 28 & $\mathrm{scl}$ & 48 & 22 & 30 & $\mathrm{scl}$ \\
\hline 2. & Lower-Kunsal & 58 & 23 & 19 & sl & 55 & 26 & 19 & sl \\
\hline 3. & Nora Garh & 56 & 25 & 19 & sl & 55 & 26 & 19 & sl \\
\hline 4. & Nora & 62 & 25 & 13 & sl & 56 & 29 & 15 & sl \\
\hline 5. & Upper-Kunsal & 54 & 28 & 18 & 1 & 50 & 29 & 21 & 1 \\
\hline & Mean & 56 & 25 & 19 & - & 53 & 26 & 21 & - \\
\hline \multicolumn{10}{|c|}{ Bhedu-Mahadev } \\
\hline 6. & Suri & 47 & 35 & 18 & 1 & 42 & 36 & 22 & 1 \\
\hline 7. & Dheera & 56 & 28 & 16 & sl & 56 & 28 & 16 & $\mathrm{sl}$ \\
\hline 8. & Panapar Kholi & 43 & 23 & 34 & $\mathrm{cl}$ & 42 & 24 & 34 & $\mathrm{cl}$ \\
\hline 9. & Bodda & 60 & 28 & 12 & sl & 53 & 28 & 19 & sl \\
\hline 10. & Mansimbal & 60 & 25 & 15 & sl & 60 & 25 & 15 & sl \\
\hline & Mean & 55 & 26 & 19 & - & 50 & 30 & 21 & - \\
\hline \multicolumn{10}{|c|}{ Bhawarna } \\
\hline 11. & Arla Khas & 58 & 24 & 18 & sl & 53 & 31 & 16 & $\mathrm{sl}$ \\
\hline 12. & Chachiyan & 57 & 27 & 16 & sl & 54 & 28 & 18 & sl \\
\hline 13. & Balla & 58 & 26 & 16 & sl & 58 & 26 & 16 & sl \\
\hline 14. & Nagri & 54 & 16 & 30 & $\mathrm{scl}$ & 54 & 16 & 30 & $\mathrm{scl}$ \\
\hline 15. & Dhakrehar & 46 & 36 & 18 & 1 & 46 & 36 & 18 & 1 \\
\hline & Mean & 53 & 30 & 17 & - & 53 & 27 & 20 & - \\
\hline \multicolumn{10}{|c|}{ Kangra } \\
\hline 16. & Kot kwal-II & 54 & 26 & 20 & scl & 54 & 26 & 20 & scl \\
\hline 17. & Kot kwal-I & 57 & 26 & 17 & sl & 56 & 28 & 16 & sl \\
\hline 18. & Zamanabad & 46 & 23 & 31 & scl & 48 & 23 & 29 & scl \\
\hline 19. & Thanpuri & 49 & 26 & 25 & $\mathrm{scl}$ & 49 & 26 & 25 & scl \\
\hline 20. & Samloti & 52 & 23 & 25 & scl & 46 & 25 & 29 & scl \\
\hline & Mean & 52 & 25 & 24 & - & 51 & 26 & 24 & - \\
\hline \multicolumn{10}{|c|}{ Dharamshala } \\
\hline 21. & Jhikli Ichi & 49 & 34 & 17 & 1 & 41 & 36 & 23 & 1 \\
\hline 22. & Lower-Bagli & 59 & 24 & 17 & sl & 55 & 26 & 19 & sl \\
\hline 23. & Upper-Bagli & 57 & 26 & 17 & sl & 53 & 28 & 19 & sl \\
\hline 24. & Tang & 44 & 28 & 28 & $\mathrm{cl}$ & 41 & 31 & 28 & $\mathrm{cl}$ \\
\hline 25. & Dadh & 59 & 25 & 16 & sl & 54 & 28 & 18 & sl \\
\hline & Mean & 52 & 28 & 20 & - & 47 & 30 & 23 & - \\
\hline \multicolumn{2}{|r|}{ Overall Range } & $43-62$ & $16-36$ & $12-34$ & sl-cl & $41-60$ & $16-36$ & $15-34$ & sl-cl \\
\hline \multicolumn{2}{|c|}{ Overall Mean $\pm S D$} & $54 \pm 5.2$ & $26 \pm 3.9$ & $20 \pm 5.6$ & - & $51 \pm 5.1$ & $27 \pm 4.1$ & $21 \pm 5.2$ & - \\
\hline
\end{tabular}

Note: scl=sandy clay loam; sl=sandy loam; cl=clay loam; l=loam 
Table.3 Bulk density $\left(\mathrm{Mg} \mathrm{m}^{-3}\right)$ under protected and conventional cultivation in district Kangra

\begin{tabular}{|c|c|c|c|c|c|c|c|}
\hline \multirow{2}{*}{$\begin{array}{l}\text { Sr. } \\
\text { No. }\end{array}$} & \multirow[t]{2}{*}{ Sites } & \multicolumn{3}{|c|}{ Protected } & \multicolumn{3}{|c|}{ Conventional } \\
\hline & & 2015 & 2016 & Mean & 2015 & 2016 & Mean \\
\hline \multicolumn{8}{|c|}{ Baijnath } \\
\hline 1. & Vikasnagar & 1.17 & 1.16 & 1.17 & 1.21 & 1.20 & 1.21 \\
\hline 2. & Lower-Kunsal & 1.24 & 1.23 & 1.24 & 1.29 & 1.28 & 1.29 \\
\hline 3. & Nora Garh & 1.17 & 1.17 & 1.17 & 1.23 & 1.21 & 1.22 \\
\hline 4. & Nora & 1.37 & 1.36 & 1.37 & 1.42 & 1.41 & 1.42 \\
\hline 5. & Upper-Kunsal & 1.18 & 1.18 & 1.18 & 1.22 & 1.24 & 1.23 \\
\hline & Mean & 1.23 & 1.22 & 1.22 & 1.27 & 1.27 & 1.27 \\
\hline \multicolumn{8}{|c|}{ Bhedu-Mahadev } \\
\hline 6. & Suri & 1.17 & 1.16 & 1.17 & 1.21 & 1.21 & 1.21 \\
\hline 7. & Dheera & 1.26 & 1.24 & 1.25 & 1.29 & 1.29 & 1.29 \\
\hline 8. & Panapar Kholi & 1.17 & 1.16 & 1.17 & 1.19 & 1.19 & 1.19 \\
\hline 9. & Bodda & 1.29 & 1.27 & 1.28 & 1.31 & 1.32 & 1.32 \\
\hline 10. & Mansimbal & 1.24 & 1.24 & 1.24 & 1.27 & 1.27 & 1.27 \\
\hline & Mean & 1.23 & 1.21 & 1.22 & 1.25 & 1.26 & 1.26 \\
\hline \multicolumn{8}{|c|}{ Bhawarna } \\
\hline 11. & Arla Khas & 1.26 & 1.25 & 1.26 & 1.28 & 1.29 & 1.29 \\
\hline 12. & Chachiyan & 1.23 & 1.23 & 1.23 & 1.25 & 1.26 & 1.26 \\
\hline 13. & Balla & 1.20 & 1.20 & 1.20 & 1.22 & 1.23 & 1.23 \\
\hline 14. & Nagri & 1.17 & 1.18 & 1.18 & 1.20 & 1.20 & 1.20 \\
\hline 15. & Dhakrehar & 1.16 & 1.15 & 1.16 & 1.24 & 1.25 & 1.25 \\
\hline & Mean & 1.20 & 1.20 & 1.20 & 1.24 & 1.25 & 1.24 \\
\hline \multicolumn{8}{|c|}{ Kangra } \\
\hline 16. & Kot kwal-II & 1.19 & 1.19 & 1.19 & 1.24 & 1.25 & 1.25 \\
\hline 17. & Kot kwal-I & 1.23 & 1.21 & 1.22 & 1.22 & 1.24 & 1.23 \\
\hline 18. & Zamanabad & 1.17 & 1.15 & 1.16 & 1.14 & 1.16 & 1.15 \\
\hline 19. & Thanpuri & 1.14 & 1.16 & 1.15 & 1.24 & 1.23 & 1.24 \\
\hline 20. & Samloti & 1.26 & 1.26 & 1.26 & 1.26 & 1.25 & 1.26 \\
\hline & Mean & 1.20 & 1.19 & 1.20 & 1.22 & 1.23 & 1.22 \\
\hline \multicolumn{8}{|c|}{$\begin{array}{l}1.19 \text { Dharamshala } \\
\end{array}$} \\
\hline 21. & Jhikli Ichi & 1.25 & 1.27 & 1.26 & 1.27 & 1.29 & 1.28 \\
\hline 22. & Lower-Bagli & 1.24 & 1.22 & 1.23 & 1.24 & 1.26 & 1.25 \\
\hline 23. & Upper-Bagli & 1.17 & 1.17 & 1.17 & 1.24 & 1.21 & 1.23 \\
\hline 24. & Tang & 1.09 & 1.08 & 1.09 & 1.13 & 1.15 & 1.14 \\
\hline 25. & Dadh & 1.22 & 1.21 & 1.22 & 1.24 & 1.24 & 1.24 \\
\hline \multirow{2}{*}{\multicolumn{2}{|c|}{$\begin{array}{c}\text { Mean } \\
\text { Overall Range }\end{array}$}} & 1.19 & 1.19 & 1.19 & 1.22 & 1.23 & 1.23 \\
\hline & & $1.09-1.37$ & $1.08-1.36$ & $1.09-1.37$ & $1.13-1.42$ & $1.15-1.41$ & $1.14-1.42$ \\
\hline \multicolumn{2}{|c|}{ Overall Mean $\pm S D$} & $1.21 \pm 0.06$ & $1.20 \pm 0.06$ & $1.21 \pm 0.06$ & $1.24 \pm 0.06$ & $1.25 \pm 0.05$ & $1.24 \pm 0.05$ \\
\hline
\end{tabular}


Table.4 Particle density $\left(\mathrm{Mg} \mathrm{m}^{-3}\right)$ under protected and conventional cultivation in district Kangra

\begin{tabular}{|c|c|c|c|c|c|c|c|}
\hline \multirow{2}{*}{$\begin{array}{l}\text { Sr. } \\
\text { No. }\end{array}$} & \multirow[t]{2}{*}{ Sites } & \multicolumn{3}{|c|}{ Protected } & \multicolumn{3}{|c|}{ Conventional } \\
\hline & & 2015 & 2016 & Mean & 2015 & 2016 & Mean \\
\hline \multicolumn{8}{|c|}{ Baijnath } \\
\hline 1. & Vikasnagar & 2.11 & 2.11 & 2.11 & 2.14 & 2.14 & 2.14 \\
\hline 2. & Lower-Kunsal & 2.36 & 2.36 & 2.36 & 2.39 & 2.39 & 2.39 \\
\hline 3. & Nora Garh & 2.24 & 2.24 & 2.24 & 2.22 & 2.20 & 2.21 \\
\hline 4. & Nora & 2.30 & 2.30 & 2.30 & 2.38 & 2.38 & 2.38 \\
\hline 5. & Upper-Kunsal & 2.39 & 2.39 & 2.39 & 2.43 & 2.44 & 2.44 \\
\hline & Mean & 2.28 & 2.28 & 2.28 & 2.31 & 2.31 & 2.31 \\
\hline \multicolumn{8}{|c|}{ Bhedu-Mahadev } \\
\hline 6. & Suri & 2.23 & 2.23 & 2.23 & 2.26 & 2.26 & 2.26 \\
\hline 7. & Dheera & 2.46 & 2.46 & 2.46 & 2.51 & 2.51 & 2.51 \\
\hline 8. & Panapar Kholi & 2.52 & 2.53 & 2.53 & 2.46 & 2.46 & 2.46 \\
\hline 9. & Bodda & 2.41 & 2.41 & 2.41 & 2.37 & 2.37 & 2.37 \\
\hline 10. & Mansimbal & 2.49 & 2.47 & 2.48 & 2.41 & 2.42 & 2.42 \\
\hline & Mean & 2.42 & 2.42 & 2.42 & 2.40 & 2.40 & 2.40 \\
\hline \multicolumn{8}{|c|}{ Bhawarna } \\
\hline 11. & Arla Khas & 2.27 & 2.27 & 2.27 & 2.27 & 2.27 & 2.27 \\
\hline 12. & Chachiyan & 2.34 & 2.33 & 2.34 & 2.31 & 2.31 & 2.31 \\
\hline 13. & Balla & 2.47 & 2.46 & 2.47 & 2.48 & 2.48 & 2.48 \\
\hline 14. & Nagri & 2.20 & 2.20 & 2.20 & 2.20 & 2.20 & 2.20 \\
\hline 15. & Dhakrehar & 2.44 & 2.44 & 2.44 & 2.47 & 2.47 & 2.47 \\
\hline & Mean & 2.34 & 2.34 & 2.34 & 2.35 & 2.35 & 2.35 \\
\hline \multicolumn{8}{|c|}{ Kangra } \\
\hline 16. & Kot kwal-II & 2.49 & 2.48 & 2.49 & 2.44 & 2.44 & 2.44 \\
\hline 17. & Kot kwal-I & 2.36 & 2.36 & 2.36 & 2.38 & 2.38 & 2.38 \\
\hline 18. & Zamanabad & 2.37 & 2.38 & 2.38 & 2.32 & 2.32 & 2.32 \\
\hline 19. & Thanpuri & 2.34 & 2.34 & 2.34 & 2.32 & 2.32 & 2.32 \\
\hline 20. & Samloti & 2.44 & 2.44 & 2.44 & 2.44 & 2.44 & 2.44 \\
\hline & Mean & 2.40 & 2.40 & 2.40 & 2.38 & 2.38 & 2.38 \\
\hline \multicolumn{8}{|c|}{ Dharamshala } \\
\hline 21. & Jhikli Ichi & 2.44 & 2.44 & 2.44 & 2.47 & 2.47 & 2.47 \\
\hline 22. & Lower-Bagli & 2.51 & 2.51 & 2.51 & 2.49 & 2.49 & 2.49 \\
\hline 23. & Upper-Bagli & 2.14 & 2.15 & 2.15 & 2.19 & 2.19 & 2.19 \\
\hline 24. & Tang & 2.43 & 2.43 & 2.43 & 2.45 & 2.45 & 2.45 \\
\hline 25. & Dadh & 2.43 & 2.43 & 2.43 & 2.43 & 2.43 & 2.43 \\
\hline & & 2.39 & 2.39 & 2.39 & 2.41 & 2.41 & 2.41 \\
\hline \multicolumn{2}{|r|}{ Overall Range } & $2.11-2.52$ & $2.15-2.53$ & $2.11-2.53$ & $2.14-2.51$ & $2.14-2.51$ & $2.14-2.51$ \\
\hline \multicolumn{2}{|c|}{ Overall Mean $\pm S D$} & $2.37 \pm 0.11$ & $2.37 \pm 0.11$ & $2.37 \pm 0.11$ & $2.37 \pm 0.10$ & $2.37 \pm 0.10$ & $2.37 \pm 0.10$ \\
\hline
\end{tabular}


Table.5 Porosity (\%) under protected and conventional cultivation in district Kangra

\begin{tabular}{|c|c|c|c|c|c|c|c|}
\hline \multirow{2}{*}{$\begin{array}{l}\text { Sr. } \\
\text { No. }\end{array}$} & \multirow[t]{2}{*}{ Sites } & \multicolumn{3}{|c|}{ Protected } & \multicolumn{3}{|c|}{ Conventional } \\
\hline & & 2015 & 2016 & Mean & 2015 & 2016 & Mean \\
\hline \multicolumn{8}{|c|}{ Baijnath } \\
\hline 1. & Vikasnagar & 44.5 & 45.0 & 44.8 & 43.5 & 43.9 & 43.7 \\
\hline 2. & Lower-Kunsal & 47.5 & 47.9 & 47.7 & 46.0 & 46.4 & 46.2 \\
\hline 3. & Nora Garh & 47.8 & 47.8 & 47.8 & 44.6 & 45.0 & 44.8 \\
\hline 4. & Nora & 40.4 & 40.9 & 40.7 & 40.3 & 40.8 & 40.5 \\
\hline 5. & Upper-Kunsal & 50.6 & 50.6 & 50.6 & 49.8 & 49.2 & 49.5 \\
\hline & Mean & 46.2 & 46.5 & 46.4 & 44.9 & 45.1 & 45.0 \\
\hline \multicolumn{8}{|c|}{ Bhedu-Mahadev } \\
\hline 6. & Suri & 47.5 & 48.0 & 47.8 & 46.5 & 46.5 & 46.5 \\
\hline 7. & Dheera & 48.8 & 49.6 & 49.2 & 48.6 & 48.6 & 48.6 \\
\hline 8. & Panapar Kholi & 53.6 & 54.2 & 53.9 & 51.6 & 51.6 & 51.6 \\
\hline 9. & Bodda & 46.5 & 47.3 & 46.9 & 44.7 & 44.3 & 44.5 \\
\hline 10. & Mansimbal & 50.2 & 49.8 & 50.0 & 47.3 & 47.5 & 47.4 \\
\hline & Mean & 49.4 & 49.8 & 49.6 & 47.8 & 47.8 & 47.8 \\
\hline \multicolumn{8}{|c|}{ Bhawarna } \\
\hline 11. & Arla Khas & 44.5 & 44.9 & 44.7 & 43.6 & 43.2 & 43.4 \\
\hline 12. & Chachiyan & 47.4 & 47.2 & 47.3 & 45.9 & 45.5 & 45.7 \\
\hline 13. & Balla & 51.4 & 51.2 & 51.3 & 50.8 & 50.4 & 50.6 \\
\hline 14. & Nagri & 46.8 & 46.4 & 46.6 & 45.5 & 45.5 & 45.5 \\
\hline 15. & Dhakrehar & 52.5 & 52.9 & 52.7 & 49.8 & 49.4 & 49.6 \\
\hline & Mean & 48.6 & 48.6 & 48.6 & 47.2 & 46.9 & 47.1 \\
\hline \multicolumn{8}{|c|}{ Kangra } \\
\hline 16. & Kot kwal-II & 52.2 & 52.0 & 52.1 & 49.2 & 48.8 & 49.0 \\
\hline 17. & Kot kwal-I & 47.9 & 48.7 & 48.3 & 48.7 & 47.9 & 48.3 \\
\hline 18. & Zamanabad & 50.6 & 51.7 & 51.2 & 50.9 & 50.0 & 50.4 \\
\hline 19. & Thanpuri & 51.3 & 50.4 & 50.9 & 46.6 & 47.0 & 46.8 \\
\hline 20. & Samloti & 48.4 & 48.4 & 48.4 & 48.4 & 48.8 & 48.6 \\
\hline & Mean & 50.1 & 50.3 & 50.2 & 48.7 & 48.5 & 48.6 \\
\hline \multicolumn{8}{|c|}{ Dharamshala } \\
\hline 21. & Jhikli Ichi & 48.8 & 48.0 & 48.4 & 48.6 & 47.8 & 48.2 \\
\hline 22. & Lower-Bagli & 50.6 & 51.4 & 51.0 & 50.2 & 49.4 & 49.8 \\
\hline 23. & Upper-Bagli & 45.3 & 45.6 & 45.5 & 43.4 & 44.7 & 44.1 \\
\hline 24. & Tang & 55.1 & 55.6 & 55.3 & 53.9 & 53.1 & 53.5 \\
\hline 25. & Dadh & 49.8 & 50.2 & 50.0 & 49.0 & 49.0 & 49.0 \\
\hline & Mean & $\mathbf{5 0 . 0}$ & 50.3 & 50.1 & 49.1 & 48.9 & 49.0 \\
\hline & Dverall Range & $40.4-55.1$ & $40.9-55.6$ & $40.7-55.3$ & 40.3-53.9 & $40.8-53.1$ & $40.5-53.5$ \\
\hline & erall Mean $\pm S D$ & $48.8 \pm 3$ & $49 \pm 3$ & $48.9 \pm 3$ & $47.4 \pm 2.9$ & $47.3 \pm 2.7$ & $47.4 \pm 2.8$ \\
\hline
\end{tabular}


Table.6 Water holding capacity (\%) under protected and conventional cultivation in district Kangra

\begin{tabular}{|c|c|c|c|c|c|c|c|}
\hline \multirow{2}{*}{$\begin{array}{l}\text { Sr. } \\
\text { No. }\end{array}$} & \multirow[t]{2}{*}{ Sites } & \multicolumn{3}{|c|}{ Protected } & \multicolumn{3}{|c|}{ Conventional } \\
\hline & & 2015 & 2016 & Mean & 2015 & 2016 & Mean \\
\hline \multicolumn{8}{|c|}{ Baijnath } \\
\hline 1. & Vikasnagar & 52.4 & 54.2 & 53.3 & 49.3 & 49.2 & 49.3 \\
\hline 2. & Lower-Kunsal & 56.1 & 57.1 & 56.6 & 49.1 & 50.2 & 49.7 \\
\hline 3. & Nora Garh & 52.2 & 53.1 & 52.7 & 48.3 & 49.1 & 48.7 \\
\hline 4. & Nora & 46.2 & 47.2 & 46.7 & 44.1 & 44.3 & 44.2 \\
\hline 5. & Upper-Kunsal & 55.1 & 54.0 & 54.6 & 51.4 & 51.3 & 51.4 \\
\hline & Mean & 52.4 & 53.1 & 52.8 & 48.4 & 48.8 & 48.6 \\
\hline \multicolumn{8}{|c|}{ Bhedu-Mahadev } \\
\hline 6. & Suri & 53.2 & 54.1 & 53.6 & 52.1 & 53.2 & 52.7 \\
\hline 7. & Dheera & 54.4 & 55.0 & 54.7 & 54.4 & 53.1 & 53.8 \\
\hline 8. & Panapar Kholi & 58.1 & 58.3 & 58.2 & 56.4 & 56.2 & 56.3 \\
\hline 9. & Bodda & 51.4 & 52.6 & 52.0 & 48.2 & 47.2 & 47.7 \\
\hline 10. & Mansimbal & 55.2 & 56.0 & 55.6 & 49.3 & 49.5 & 49.4 \\
\hline & Mean & 54.5 & 55.2 & 54.8 & 52.1 & 51.8 & 52.0 \\
\hline \multicolumn{8}{|c|}{ Bhawarna } \\
\hline 11. & Arla Khas & 48.0 & 48.6 & 48.3 & 47.2 & 47.3 & 47.3 \\
\hline 12. & Chachiyan & 51.0 & 51.4 & 51.2 & 49.1 & 48.2 & 48.7 \\
\hline 13. & Balla & 56.1 & 56.2 & 56.2 & 54.1 & 55.4 & 54.8 \\
\hline 14. & Nagri & 51.1 & 51.3 & 51.2 & 49.2 & 49.5 & 49.4 \\
\hline 15. & Dhakrehar & 58.0 & 58.0 & 58.0 & 52.3 & 53.5 & 52.9 \\
\hline & Mean & 52.8 & 53.1 & 53.0 & 50.4 & 50.8 & 50.6 \\
\hline \multicolumn{8}{|c|}{ Kangra } \\
\hline 16. & Kot kwal-II & 58.4 & 58.0 & 58.2 & 54.5 & 53.2 & 53.9 \\
\hline 17. & Kot kwal-I & 52.2 & 52.5 & 52.4 & 53.5 & 52.4 & 52.9 \\
\hline 18. & Zamanabad & 53.4 & 54.0 & 53.7 & 53.4 & 52.1 & 52.8 \\
\hline 19. & Thanpuri & 57.1 & 56.2 & 56.7 & 51.0 & 52.2 & 51.6 \\
\hline 20. & Samloti & 51.4 & 51.0 & 51.2 & 53.0 & 53.4 & 53.2 \\
\hline & Mean & 54.5 & 54.3 & 54.4 & 53.1 & 52.7 & 52.9 \\
\hline \multicolumn{8}{|c|}{ Dharamshala } \\
\hline 21. & Jhikli Ichi & 52.0 & 52.0 & 52.0 & 52.2 & 52.6 & 52.4 \\
\hline 22. & Lower-Bagli & 54.1 & 55.0 & 54.6 & 54.5 & 52.1 & 53.3 \\
\hline 23. & Upper-Bagli & 48.2 & 49.4 & 48.8 & 48.0 & 49.2 & 48.6 \\
\hline 24. & Tang & 60.3 & 60.2 & 60.3 & 58.4 & 56.2 & 57.3 \\
\hline 25. & Dadh & 53.2 & 54.0 & 53.6 & 52.2 & 52.1 & 52.2 \\
\hline \multirow{2}{*}{\multicolumn{2}{|c|}{$\begin{array}{c}\text { Mean } \\
\text { Overall Range }\end{array}$}} & 53.6 & 54.1 & 53.8 & 53.1 & 52.4 & 52.7 \\
\hline & & $46.2-60.3$ & $47.2-60.2$ & $46.7-60.3$ & $44.1-58.4$ & $44.3-56.2$ & $44.2-57.3$ \\
\hline \multicolumn{2}{|c|}{ Overall Mean $\pm S D$} & $53.6 \pm 3.2$ & $54 \pm 3.0$ & $53.8 \pm 3.1$ & $51.4 \pm 3.1$ & $51.3 \pm 2.8$ & $51.3 \pm 2.9$ \\
\hline
\end{tabular}


Table.7 Mean weight diameter $(\mathrm{mm})$ under protected and conventional cultivation in district Kangra

\begin{tabular}{|c|c|c|c|c|c|c|c|}
\hline \multirow[t]{2}{*}{ Sr. No. } & \multirow[t]{2}{*}{ Sites } & \multicolumn{3}{|c|}{ Protected } & \multicolumn{3}{|c|}{ Conventional } \\
\hline & & 2015 & 2016 & Mean & 2015 & 2016 & Mean \\
\hline \multicolumn{8}{|c|}{ Baijnath } \\
\hline 1. & Vikasnagar & 2.22 & 2.24 & 2.23 & 2.02 & 2.03 & 2.03 \\
\hline 2. & Lower-Kunsal & 1.82 & 1.83 & 1.83 & 1.44 & 1.46 & 1.45 \\
\hline 3. & Nora Garh & 1.40 & 1.41 & 1.41 & 1.36 & 1.37 & 1.37 \\
\hline 4. & Nora & 1.26 & 1.27 & 1.27 & 1.04 & 1.03 & 1.04 \\
\hline 5. & Upper-Kunsal & 1.67 & 1.64 & 1.66 & 1.19 & 1.19 & 1.19 \\
\hline & Mean & 1.67 & 1.68 & 1.68 & 1.41 & 1.42 & 1.41 \\
\hline \multicolumn{8}{|c|}{ Bhedu-Mahadev } \\
\hline 6. & Suri & 1.37 & 1.38 & 1.38 & 1.36 & 1.37 & 1.37 \\
\hline 7. & Dheera & 1.42 & 1.44 & 1.43 & 1.47 & 1.45 & 1.46 \\
\hline 8. & Panapar Kholi & 1.69 & 1.68 & 1.69 & 1.56 & 1.57 & 1.57 \\
\hline 9. & Bodda & 1.14 & 1.16 & 1.15 & 1.09 & 1.08 & 1.09 \\
\hline 10. & Mansimbal & 1.07 & 1.06 & 1.07 & 1.03 & 1.04 & 1.04 \\
\hline & Mean & 1.34 & 1.34 & 1.34 & 1.30 & 1.30 & 1.30 \\
\hline \multicolumn{8}{|c|}{ Bhawarna } \\
\hline 11. & Arla Khas & 1.32 & 1.34 & 1.33 & 1.24 & 1.26 & 1.25 \\
\hline 12. & Chachiyan & 1.52 & 1.54 & 1.53 & 1.48 & 1.44 & 1.46 \\
\hline 13. & Balla & 2.21 & 2.22 & 2.22 & 2.14 & 2.12 & 2.13 \\
\hline 14. & Nagri & 1.27 & 1.26 & 1.27 & 1.21 & 1.22 & 1.22 \\
\hline 15. & Dhakrehar & 2.14 & 2.16 & 2.15 & 2.16 & 2.14 & 2.15 \\
\hline & Mean & 1.69 & 1.70 & 1.70 & 1.65 & 1.64 & 1.64 \\
\hline \multicolumn{8}{|c|}{ Kangra } \\
\hline 16. & Kot kwal-II & 1.31 & 1.33 & 1.32 & 1.22 & 1.23 & 1.23 \\
\hline 17. & Kot kwal-I & 1.03 & 1.02 & 1.03 & 1.04 & 1.01 & 1.03 \\
\hline 18. & Zamanabad & 1.18 & 1.19 & 1.19 & 1.28 & 1.27 & 1.28 \\
\hline 19. & Thanpuri & 1.47 & 1.46 & 1.47 & 1.39 & 1.38 & 1.39 \\
\hline 20. & Samloti & 1.06 & 1.08 & 1.07 & 1.04 & 1.02 & 1.03 \\
\hline & Mean & 1.21 & 1.22 & 1.21 & 1.19 & 1.18 & 1.19 \\
\hline \multicolumn{8}{|c|}{ Dharamshala } \\
\hline 21. & Jhikli Ichi & 1.23 & 1.24 & 1.24 & 1.07 & 1.04 & 1.06 \\
\hline 22. & Lower-Bagli & 1.59 & 1.57 & 1.58 & 1.44 & 1.41 & 1.43 \\
\hline 23. & Upper-Bagli & 1.63 & 1.64 & 1.64 & 1.84 & 1.83 & 1.84 \\
\hline 24. & Tang & 2.20 & 2.24 & 2.22 & 2.15 & 2.14 & 2.15 \\
\hline 25. & Dadh & 1.08 & 1.09 & 1.09 & 1.06 & 1.08 & 1.07 \\
\hline & & 1.55 & 1.56 & 1.55 & 1.51 & 1.50 & 1.51 \\
\hline \multicolumn{2}{|r|}{ Overall Range } & $1.03-2.22$ & $1.02-2.24$ & $1.03-2.23$ & $1.03-2.16$ & $1.01-2.14$ & $1.03-2.15$ \\
\hline \multicolumn{2}{|c|}{ Overall Mean $\pm S D$} & $1.49 \pm 0.36$ & $1.50 \pm 0.36$ & $1.49 \pm 0.36$ & $1.41 \pm 0.35$ & $1.40 \pm 0.35$ & $1.41 \pm 0.35$ \\
\hline
\end{tabular}


Figure.1 Locations of soil samples

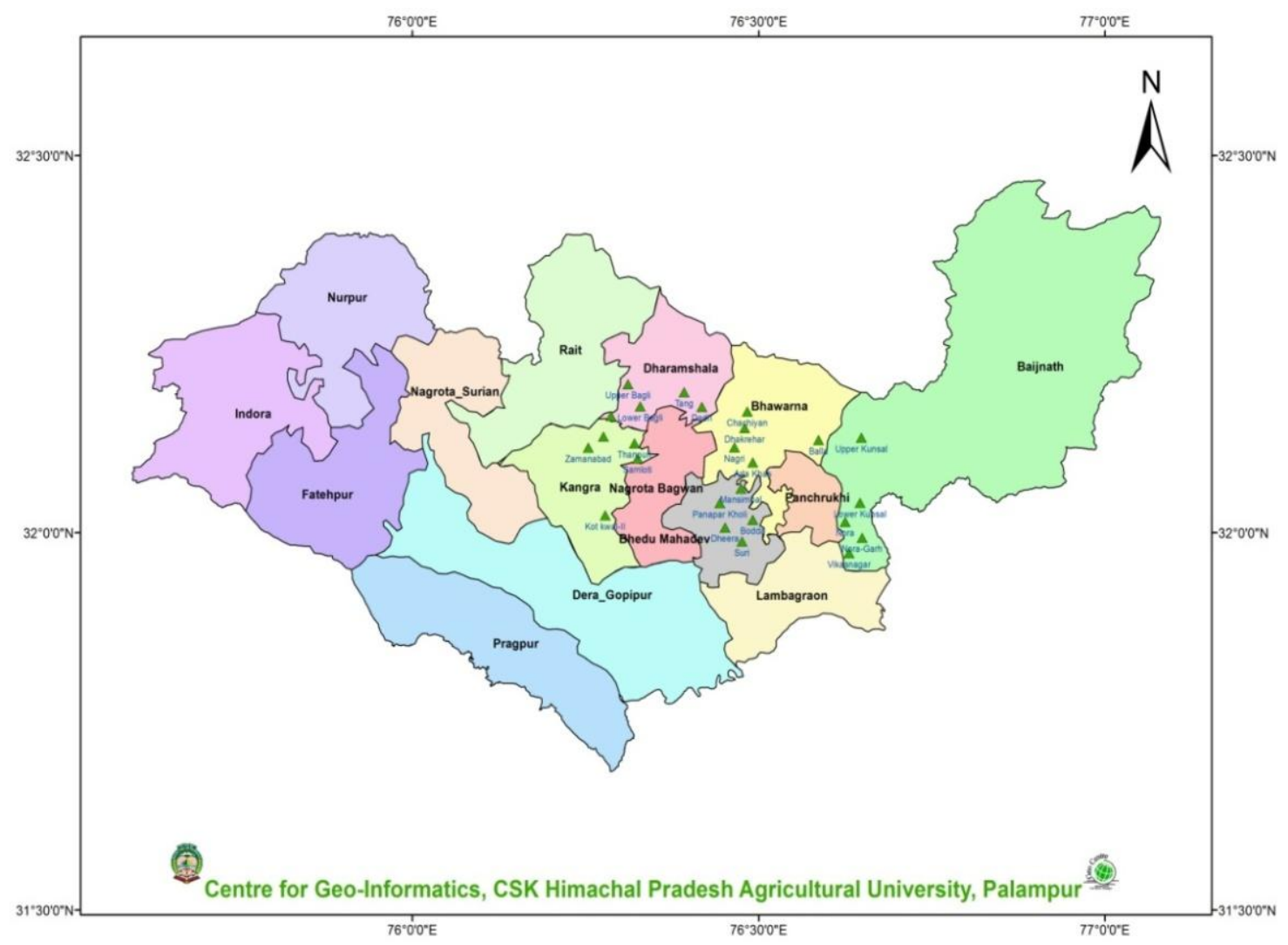

The highest mean water holding capacity of a block as a whole under the conventional system was observed in Kangra (52.9\%), while, the lowest was observed for Baijnath block (48.6\%).Water holding capacity in soil is influenced by many factors especially soil texture, organic matter and structure of the soil.

The variation observed between different sites under protected and open environment, might be the consequence of variation in management practices followed by the farmers. Increase in water holding capacity under the protected production system might be attributed to stable aggregates and better structure as compared to open conditions of vegetable production. Whereas, comparatively lower values under open environment might be due to the low stability of aggregates as well repeated intensive tillage practices and exposure of the organic matter to oxidation.

\section{Mean Weight Diameter (MWD)}

Water-stable aggregates larger than $2 \mathrm{~mm}$ are the most important fractions in assessing the effects of fertilization practices on soil aggregation, for they exert a strong influence on the mean weight diameter, a comprehensive index for evaluating soil structure (Angers and Mehuys 1993). The MWD in the present investigation for 2015 and 2016 under protected conditions varied from 1.03 to $2.22 \mathrm{~mm}$ and 1.02 to $2.24 \mathrm{~mm}$, respectively. Considering mean values across all the locations for 2015 and 2016, MWD was $1.49 \pm 0.36 \mathrm{~mm}$ and $1.50 \pm 0.36 \mathrm{~mm}$, respectively (Table 7). Among different blocks under protected system of vegetable production, Bhawarna recorded the highest 
MWD (1.70 $\mathrm{mm})$ under polyhouse soils, while Kangra block had the lowest average MWD (1.21 mm).

Under conventional vegetable cultivation it varied from $1.03-2.16 \mathrm{~mm}$ and 1.01 to 2.14 $\mathrm{mm}$ for 2015 and 2016, respectively. Overall mean values of MWD across all the locations for 2015 and 2016 were $1.41 \pm 0.35 \mathrm{~mm}$ and $1.40 \pm 0.35 \mathrm{~mm}$, respectively conventional system of vegetable production followed almost similar trend as per polyhouse soil and found highest and lowest values of MWD under similar blocks as observed under protected conditions. Comparatively higher MWD values observed under protected system might be due to tillage practices which were carried out within the range of optimum moisture conditions under protected cultivation of vegetables ensuring the least destruction of soil aggregates. As excessive tilling of soil reduce the organic matter through oxidation and erosion of soil hence reduce aggregate stability. Further, soil under protected condition remains protected from the beating action of rain and erosion. Frequent addition of organic matter is another reason for better stable aggregates under protected condition as compared to open soil. Polyhouse growers are more concerned about the proper management practices under polyhouse cultivation owing to lesser area and higher productivity and net returns. A strong influence from the addition of organic matter on the stability of aggregates was seen, and this was more evident in the polyhouse. Also the sites under protected system where practice of lime addition was followed higher MWD was recorded, because calcium act as a binding agent in formation of large sized aggregates as calcium is a flocculating agent. Calcium ions associated with clay generally promote aggregation and thus MWD. Chaudhary et al., (2005) and Khan (2015) have similar findings of calcium as stabilization and binding agent and also reported similar results which confirmed the present findings.

In conclusions, the frequent use of organic manures resulted in lower bulk density under polyhouse conditions as compared to conventional open condition. Continuous organic fertilization indicated that the use of FYM and organic manures produced a decrease in the BD and therefore an increase in the aggregate stability of soil. Aggregate stability values were higher in the greenhouse than in the outdoor plots. Therefore differences in management practices exert a significant influence on the evolution of physical properties. This study indicated that the comparatively better management practices followed by farmers in chase of better returns resulted in an increase in the soil organic matter, depending upon different management practices followed by different farmers. The use of sustainable management techniques such as the use of organic amendments and low or no tillage improved soil physical properties.

\section{References}

Blake, G.R. and Hartge, K.H. 1986. Bulk Density. In Methods of Soil Analysis. Part I. Physical and Mineralogical Methods (A Klute, eds.). American Society of Agronomy: Madison, Wisconsin; Agronomy Monograph 9: 364-367

Benbi, D.K and Saroa, G.S. 2012. Soil health and agriculture sustainability in Punjab. In: $77^{\text {th }}$ Annual Convention of Indian Society of Soil Science, Dept. of Soil Science, PAU, Ludhiana

Chander, G., Sharma, S., Sharma, V. and Verma, S. 2014. Micronutrient cations status in vegetable growing soils of subhumid and wet temperate zones of Himachal Pradesh. Himachal Journal of Agricultural Research 40(1): 79-83 
Chaudhary, P.R., Dodha, V., Ahire, V.D., Chkravarty, M. and Maity, S. 2005. Soil bulk density as related to soil texture, organic matter content and available total nutrients of Coimbatore soil. International Journal of Scientific and Research Publications 3(2): 1-7

Doran, J. Wand Parkin T.B. 1994. Defining and assessing soil quality. In: Defining Soil Quality for Sustainable Environment. Soil Science Society of America. Publication no. 35. Madison, WI. Pp 3-21

Gupta, R.P., and Dhakshinamoorthy, C. 1980. Procedures for Physical Analysis of Soils and collection of Agrometerological Data, Division of Agricultural Physics, Indian Agricultural Research Institute, New Delhi

Herencia, J.F., Galavis-Garcia, P.A., and Maqueda, C. 2011. Long term effect of organic and mineral fertilization on soil physical properties under greenhouse and outdoor management practices. Pedosphere 21: 443-453

Hillel, D. 1980. Fundamentals of soil physics. Harcourt Brace Jovanivich Publisher.
Academic Press, Inc. San Diego. 413 p

Khan, M., A. 2015. Effect of lime and fly ash on cation exchange capacity and unconfined compressive strength (UCS) of soils. M.Tech. Thesis, NIT Rourkela, Odisha

Kyandiah, R. 2012. Impact of different land uses on runoff and nutrient losses in Ga3 a micro watershed of Giri river in Solan district of Himachal Pradesh. M.Sc Thesis, p 74 Department of Soil Science and Water Management, UHF Nauni, Solan, India

Piper, C.D., 1950. Soil and Plant Analysis. Inc. Sci. Pub. INC, New York

Piper, C.S., 1966. Soil and Plant Analysis (Asian edition). Hans Publisher, Bombay. p 223-237

Reynolds, W.D., Drury, C.F., Tan, C.S., Fox, C.A., and Yang, X.M. 2009. Use of indicators and pore volume function characteristics to quantify soil physical quality. Geoderma 152: 252-263

Yoder, R.E. 1936. A direct method of aggregate analysis and study of the physical nature of erosion losses. Journal of American Society of Agronomy 28: 337-351

\section{How to cite this article:}

Shabnam and Sanjay K. Sharma. 2018. Soil Physical Properties as Influenced with Protected and Conventional Vegetable Production System in District Kangra of Himachal Pradesh. Int.J.Curr.Microbiol.App.Sci. 7(11): 3503-3516. doi: https://doi.org/10.20546/ijcmas.2018.711.400 